Editors' Note: In WriteClick this week, Dr. Taschner points out that the ASPHD1 variant described in the article "Novel CLN3 mutation causing autophagic vacuolar myopathy" as "one 2-base pairs deletion (c.515_516insTGG:p. Gly172delinsGlyGly)" should have been described as a duplication: c.513_515dup resulting in p.(Gly172dup), which might retain some functionality. The authors agree and a correction to the original article has been issued. Drs. Beardsley and Le discuss possible biases in "Antiretroviral penetration into the CNS and incidence of AIDS-defining neurologic conditions."

—Megan Alcauskas, MD, and Robert C. Griggs, $M D$

\section{NOVEL CLN3 MUTATION CAUSING AUTOPHAGIC} VACUOLAR MYOPATHY

Peter E. Taschner, Leiden, Netherlands: Cortese et al. described the ASPHD1 variant found as "one 2-base pairs deletion (c.515_516insTGG:p.Gly172delinsGlyGly)," which is incorrect. The HGVS part suggests a 3-bp insertion, which should have been described as a duplication, c.513_515dup resulting in p.(Gly172dup), which might retain some functionality. If nucleotides 515 and 516 were deleted and replaced by TGG, the correct description should be c.515_516delinsTGG predicted to result in a likely deleterious frameshift: p.(Gly172Valfs*154). Because the authors cannot exclude any effect of homozygosity for the ASPHDI variant on the phenotypes of the patients, a clearer description would help the reader correctly assess the results.

Author Response: Andrea Cortese, Pavia, Italy; Arianna Tucci, London: The authors thank Dr. Taschner for his response and apologize for the mistake regarding the ASPHD1 variant. ${ }^{1}$ The ASPHD1 variant should have been described as a duplication, c.513_515dup resulting in p.(Gly172dup), which might retain some functionality.

This is a known issue with the software used to annotate the variants in the whole exome sequencing data.

Editor's Note: A Correction appears on page 633 of this issue related to this WriteClick exchange.

(C) 2015 American Academy of Neurology
1. Cortese A, Tucci A, Piccolo G, et al. Novel CLN3 mutation causing autophagic vacuolar myopathy. Neurology 2014; 82:2072-2076.

\section{ANTIRETROVIRAL PENETRATION INTO THE CNS AND INCIDENCE OF AIDS-DEFINING NEUROLOGIC CONDITIONS}

Justin Beardsley, Thuy Le, Ho Chi Minh City, Vietnam: Caniglia et al. ${ }^{1}$ furthered the debate on the clinical significance of the CNS penetration effectiveness (CPE) score. The authors and the editorialists $^{2}$ cautioned against selecting high-CPE regimens -often with less-favorable dosing and side-effect profiles-for patients with HIV-associated neurologic disorders. No high-CPE regimens are recommended by the WHO for first-line therapy. ${ }^{3}$ Although a lowcost combination regimen of nevirapine and zidovudine is widely used in developing countries, the risks of this treatment are unclear.

Selection bias and confounders can undermine observational cohort studies. The authors never mentioned the bias evident when physicians avoid efavirenz in patients with neuropsychiatric issues. These patients would receive regimens with coincidentally higher CPEs but may already have higher risks for developing AIDS dementia. This diagnosis of exclusion takes time. Even the sensitivity analysis excluding cases of AIDS dementia diagnosed within 1 year of enrollment may not address this confounder.

Almost $70 \%$ of patients changed regimen in this study, further challenging its validity. Although reflecting the reality of multiple treatment choices in high-income countries, it calls for careful interpretation of the findings. Continued prospective work is needed to elucidate the clinical significance of the CPE score.

Author Response: Ellen C. Caniglia, Miguel A. Hernan, on behalf of the HIV-CAUSAL Collaboration, Boston: Drs. Beardsley and Le offer a possible explanation for the association we found between initiating an antiretroviral regimen with a high CPE score and the incidence of HIV dementia. ${ }^{1}$ They noted the bias evident when physicians avoid prescribing efavirenz to individuals with neurocognitive 


\begin{tabular}{|c|c|c|c|c|c|c|c|c|}
\hline \multirow[t]{2}{*}{ Table } & \multicolumn{8}{|c|}{ HIV dementia hazard ratios for CPE score, HIV-CAUSAL collaboration, 1998-2013 } \\
\hline & \multicolumn{2}{|c|}{ Person-years } & \multicolumn{2}{|c|}{ No. of events } & \multicolumn{2}{|c|}{ Unadjusted hazard ratio $(95 \% \mathrm{Cl})$} & \multicolumn{2}{|c|}{ IPW-adjusted hazard ratio $(95 \% \mathrm{Cl})$} \\
\hline CPE score & Overall & Excluding efavirenz & Overall & Excluding efavirenz & Overall & Excluding efavirenz & Overall & Excluding efavirenz \\
\hline Low & 140,962 & 82,323 & 127 & 95 & 1.00 (Reference) & 1.00 (Reference) & 1.00 (Reference) & 1.00 (Reference) \\
\hline Medium & 86,799 & 46,219 & 72 & 46 & $0.97(0.72,1.30)$ & $0.98(0.68,1.40)$ & $1.01(0.73,1.39)$ & $1.04(0.72,1.50)$ \\
\hline High & 32,097 & 28,930 & 36 & 34 & $1.55(1.06,2.26)$ & $1.33(0.89,1.98)$ & $1.74(1.15,2.65)$ & $1.80(1.10,2.95)$ \\
\hline
\end{tabular}

Abbreviations: $\mathrm{Cl}=$ confidence interval; $\mathrm{CPE}=\mathrm{CNS}$ penetration effectiveness; IPW = inverse probability weighting.

symptoms or neuropsychiatric issues. Efavirenz is commonly used as part of the regimens with low and medium CPE scores. However, an analysis that excludes individuals initiating a regimen with efavirenz does not materially affect our results (table). We agree with Beardsley and Le that future studies are needed to examine the effect of CNS penetration on the incidence of HIV dementia.

(c) 2015 American Academy of Neurology
1. Caniglia EC, Cain LE, Justice A, et al. Antiretroviral penetration into the CNS and incidence of AIDS-defining neurologic conditions. Neurology 2014;83:134-141.

2. Berger JR, Clifford DB. The relationship of CPE to HIV dementia: slain by an ugly fact? Neurology 2014;83:109110 .

3. World Health Organization. March 2014 supplement to the 2013 consolidated guidelines on the use of antiretroviral drugs for treating and preventing HIV infection. Available at: www. who.int/hiv/pub/guidelines/arv2013/arvs2013upplement_ march2014/en/. Accessed July 22, 2014.

\section{CORRECTION}

Novel CLN3 mutation causing autophagic vacuolar myopathy

In the article "Novel CLN3 mutation causing autophagic vacuolar myopathy" by A. Cortese et al. (Neurology ${ }^{\circledR}$ 2014;82:2072-2076), there is an error in the Results regarding the ASPHD1 variant. The ASPHD1 variant should have been described as a duplication: c.513_515dup resulting in p.(Gly172dup), which might retain some functionality (this is a known issue with the software used to annotate the variants in the whole exome sequencing data). The authors regret the error. 


\section{Neurology}

\section{Antiretroviral penetration into the CNS and incidence of AIDS-defining neurologic conditions}

Justin Beardsley, Ellen C. Caniglia, Thuy Le, et al.

Neurology 2015;84;632-633

DOI 10.1212/01.wnl.0000461261.51494.39

This information is current as of February 9, 2015

Updated Information \& Services

References

Permissions \& Licensing

Reprints including high resolution figures, can be found at: http://www.neurology.org/content/84/6/632.2.full.html

This article cites 2 articles, 2 of which you can access for free at: http://www.neurology.org/content/84/6/632.2.full.html\#\#ref-list-1

Information about reproducing this article in parts (figures,tables) or in its entirety can be found online at:

http://www.neurology.org/misc/about.xhtml\#permissions

Information about ordering reprints can be found online: http://www.neurology.org/misc/addir.xhtml\#reprintsus

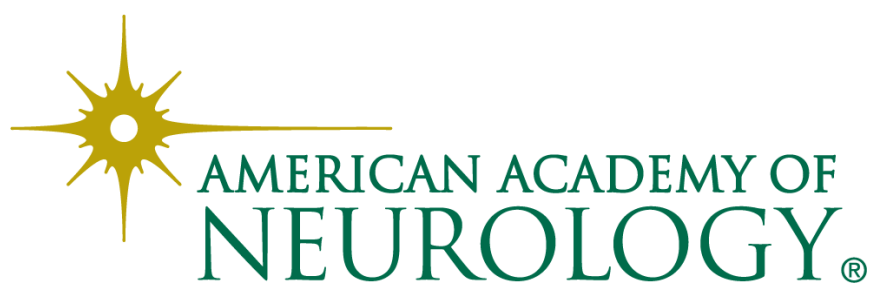

